Supporting Information for:

\title{
The Molecular Path Approaching the Active Site in Catalytic Metal- Organic Frameworks
}

Ana E. Platero-Prats, ${ }^{1}$ Andreas Mavrandonakis, ${ }^{2}$ Jian Liu, ${ }^{3}$ Zhihengyu Chen, ${ }^{4}$ Zhijie Chen, ${ }^{3}$ Zhanyong Li, ${ }^{3}$ Andrey A. Yakovenko, ${ }^{1}$ Leighanne C. Gallington, ${ }^{1}$ Joseph T. Hupp, ${ }^{3}$ Omar K. Farha, ${ }^{3,5}$ Christopher J. Cramer, ${ }^{2}$ Karena W. Chapman ${ }^{1,4 *}$

${ }^{1}$ X-ray Science Division, Advanced Photon Source, Argonne National Laboratory, Argonne, Illinois 60439, United States

${ }^{2}$ Department of Chemistry, Chemical Theory Center, and Supercomputing Institute, University of Minnesota, MN 55455, USA

${ }^{3}$ Department of Chemistry, Northwestern University, Evanston, Illinois 60208, United States

${ }^{4}$ Department of Chemistry, Stony Brook University, Stony Brook, New York, 11790, United States

${ }^{5}$ Department of Chemical and Biological Engineering, Northwestern University, Evanston, Illinois 60208, United States

\section{Table of Contents}

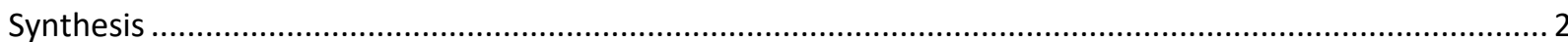

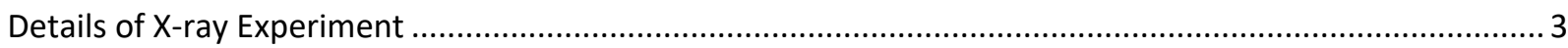

Temperature- \& guest-dependent lattice dimensions ....................................................................

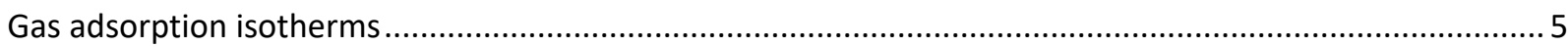

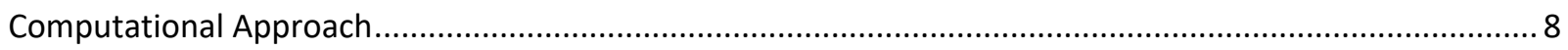

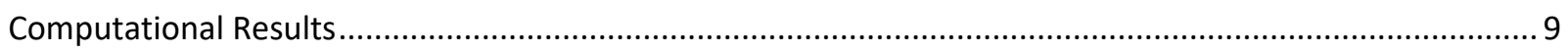

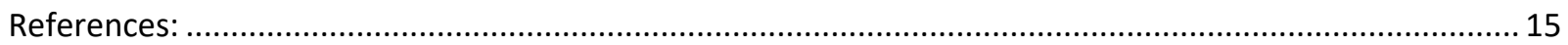




\section{Synthesis}

NU-1000. The NU-1000 was synthesized through a modified literature procedure. ${ }^{1} \mathrm{~A}$ screw cap jar $(1.0 \mathrm{~L})$ was charged with $\mathrm{ZrOCl}_{2} .8 \mathrm{H}_{2} \mathrm{O}(4.85 \mathrm{~g}, 27.3 \mathrm{mmol})$, benzoic acid $(135.0 \mathrm{~g}, 1.1 \mathrm{~mol})$ and DMF $(300 \mathrm{~mL})$, forming a suspension, which was sonicated until clear. Separately, a sample of $\mathrm{H}_{4}$ TBAPy $(2.0 \mathrm{~g}, 3.0 \mathrm{mmol})$ and $100 \mathrm{~mL}$ of DMF were added to a $250 \mathrm{~mL}$ screw cap jar, resulting in a yellow suspension. Both jars were heated in an oven at $100^{\circ} \mathrm{C}$, after the solutions were mixed and heated at $120^{\circ} \mathrm{C}$ for $16 \mathrm{~h}$. The supernatant was decanted while hot. DMF $(250 \mathrm{~mL})$ was added to the container to wash the solid sample and the yellow crystalline samples were harvested via centrifugation. The samples were washed three times.

Activation. The as-synthesized NU-1000 was suspended in DMF (400 mL) in a screw cap jar $(1.0 \mathrm{~L})$ and 8 $\mathrm{M} \mathrm{HCl}(25 \mathrm{~mL})$ was added. The jar was heated at $120^{\circ} \mathrm{C}$ for $16 \mathrm{~h}$. The supernatant liquid was decanted while hot. DMF $(250 \mathrm{~mL})$ was added to the container to wash the solid sample and the yellow crystalline samples were harvested via centrifugation. The solid was washed three times with 15 min between washings. The solid was suspended in DMF $(400 \mathrm{~mL})$ for $8 \mathrm{~h}$, then washed 3 times with acetone $(250 \mathrm{~mL})$. Subsequently, the solid was suspended in acetone for $24 \mathrm{~h}$. NU-1000 was obtained via centrifugation and placed in a vacuum oven set at $100{ }^{\circ} \mathrm{C}$ for $1 \mathrm{~h}$, before being heated under a dynamic vacuum at $120^{\circ} \mathrm{C}$ for $24 \mathrm{~h}$.

Synthesis of Ni-Facac, Ni- NU1000 via Atomic Layer Deposition (ALD). Facac was pulsed following the time sequence of $t_{1}-t_{2}-t_{3}$, where $t_{1}$ is the precursor pulse time, $t_{2}$ the exposure time, and $t_{3}$ the $N_{2}$ purge time $\left(t_{1}=0.015 \mathrm{~s}, t_{2}=t_{3}=300 \mathrm{~s}\right)$. To ensure the Facac saturates the active sites on $\mathrm{Zr}_{6}$ nodes, 100 Facac pulses were applied on $60.0 \mathrm{mg}$ of NU-1000.

The Ni-ALD step followed reported procedures. $^{2}$ The compound bis $\left(\mathrm{N}, \mathrm{N}^{\prime}\right.$-ditert-butylacetamidinato)nickel(II), $\mathrm{Ni}\left(\mathrm{MeC}(\mathrm{Nt}-\mathrm{Bu})_{2}\right)_{2}$ and deionized $\mathrm{H}_{2} \mathrm{O}$ were used to form $\mathrm{NiO}_{x} \mathrm{H}_{\mathrm{y}}$ clusters on the $\mathrm{Zr}_{6}$ nodes. In a typical experiment, a custom-made stainless-steel powder sample holder containing microcrystalline Facac-AIM-NU-1000 (60.0 mg, $0.028 \mathrm{mmol}$ ) was placed in the ALD chamber, which was held at $125{ }^{\circ} \mathrm{C}$ for $30 \mathrm{~min}$ to remove physisorbed water before dosing with the Ni precursor. A cylinder containing $\mathrm{Ni}\left(\mathrm{MeC}(\mathrm{Nt}-\mathrm{Bu})_{2}\right)_{2}$ was held at $120^{\circ} \mathrm{C}$, and each of its pulses followed the time sequence of $t_{1}-t_{2}-t_{3}\left(t_{1}=1 \mathrm{~s}, t_{2}=t_{3}=300 \mathrm{~s}\right)$. To ensure full metalation of the $Z r_{6}$ sites throughout the microcrystals, the $\mathrm{Ni}\left(\mathrm{MeC}(\mathrm{Nt}-\mathrm{Bu})_{2}\right)_{2}$ pulsing cycle was run 100 times before subjecting the MOF to $\mathrm{H}_{2} \mathrm{O}$ pulses $\left(t_{1}=0.015 \mathrm{~s}\right.$, $\left.t_{2}=t_{3}=300 \mathrm{~s}\right)$. The prepared material, either Ni-Facac or Ni-NU1000, was stored in a vacuum desiccator. The $\mathrm{Ni}$ within the materials were quantified using ICP-AES as $3.4 \pm 0.3$ and $4.0 \pm 0.3 \mathrm{Ni}$ per $\mathrm{Zr}_{6}$ node for $\mathrm{Ni}$ Facac and Ni-NU1000, respectively. 


\section{Details of X-ray Experiment}

In situ synchrotron PXRD data $(\lambda=0.2114 \AA$ ) were collected for Ni-oxo clusters supported on NU-1000 ( $\mathrm{Ni}$ NU-1000), supported on Facac-modified NU-1000 (Ni-Facac), and Ni-free NU-1000 at beamline 11-ID-B at the Advanced Photon Source, at Argonne National Laboratory. Capillary-loaded samples were assembled into flow cell reactor that allows controlled gas atmosphere and temperature. ${ }^{3}$ To evaluate the gasbinding locations following activation at $200{ }^{\circ} \mathrm{C}$ in $3.5 \% \mathrm{H}_{2}$ in $\mathrm{He}$, data were collected under ethylene, ethane and $3.5 \% \mathrm{H}_{2}$ in $\mathrm{He}$ gas flow at $10^{\circ} \mathrm{C}$ intervals from 40 and $100{ }^{\circ} \mathrm{C}$. Additional diffraction data were collected for Ni-free Facac-modified NU-1000 (Facac-NU1000) under 3.5\% $\mathrm{H}_{2}$ in He gas flow were collected X-ray scattering images were reduced to 1D diffraction data as a function of scattering angle within gsas- $1 I^{4}$. The lattice parameters and peak intensities were evaluated using Le Bail fits to the data within Jana2006 ${ }^{5}$. Changes in the peak intensities observed for different gases and at different temperatures reflect differences in the distribution of adsorbed guest molecules and their associated electron density. Structure envelopes were constructed based on the peak intensities, ${ }^{6-7}$ and the electron density associated with the adsorbed guests visualized as the difference envelope density (DED). ${ }^{8-9}$ The DEDs were obtained by subtracting the structure envelope for the corresponding guest-free system measured under $3.5 \% \mathrm{H}_{2}$ in $\mathrm{He}$ at intermediate temperature $\left(70^{\circ} \mathrm{C}\right) .{ }^{9}$

DEDs to locate the principal electron density associated with the $\mathrm{Ni}$-oxo clusters and Facac-ligands were calculated based on the guest-free structure envelopes obtained for Ni-Facac, Ni-NU1000, NU1000 and Facac-NU1000. The additional electron density associated with the Facac functionalization (Structure envelope (Facac-NU100)- Structure envelope (NU1000)) is in the hexagonal pore, next to the $\mathrm{Zr}_{6}$-node. The additional electron density associated with the $\mathrm{Ni}$-oxo functionalization is in the same location for both NU-1000 and Facac-NU1000; in the small pore, bridging adjacent $\mathrm{Zr}_{6}$ nodes, as has been documented previously. ${ }^{10}$
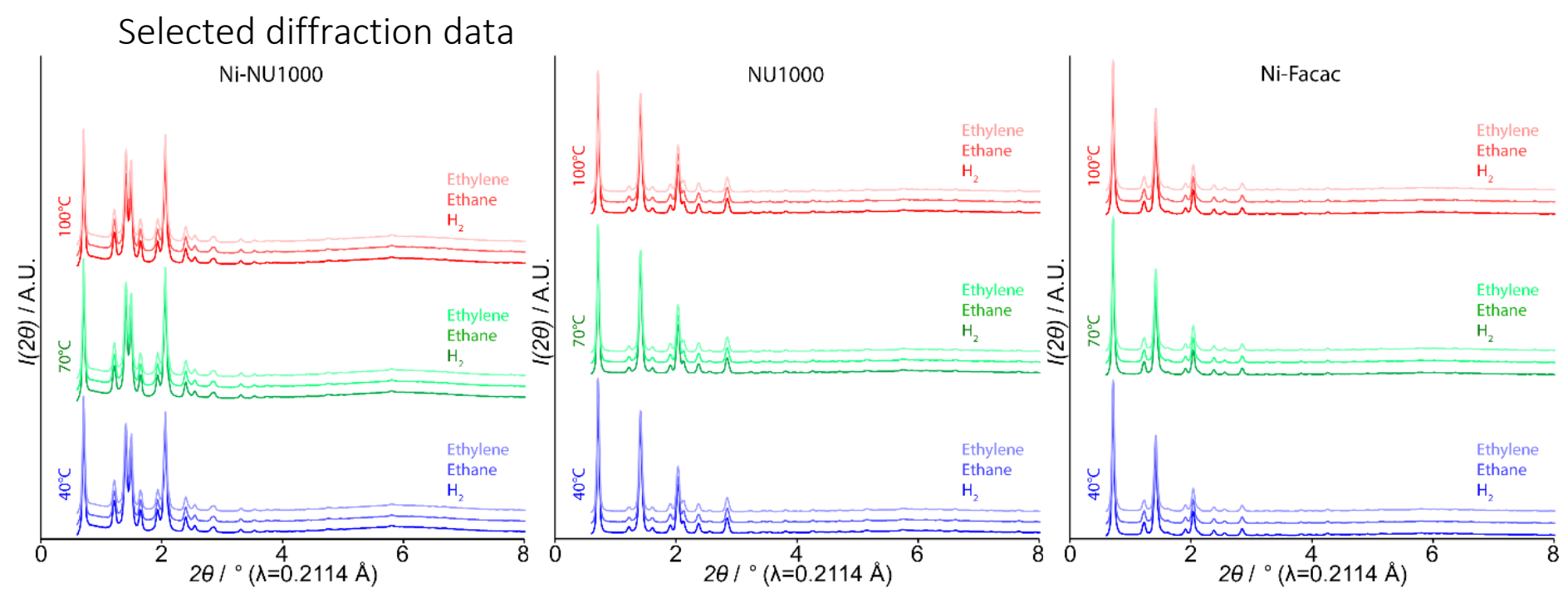

Figure S1. XRD of NU1000, Ni-NU1000, and Ni-Facac at 40,70, and $100^{\circ} \mathrm{C}$ and $\mathrm{H}_{2}$, ethane, and ethylene atmospheres 
Temperature- \& guest-dependent lattice dimensions Ni-NU1000 NU1000
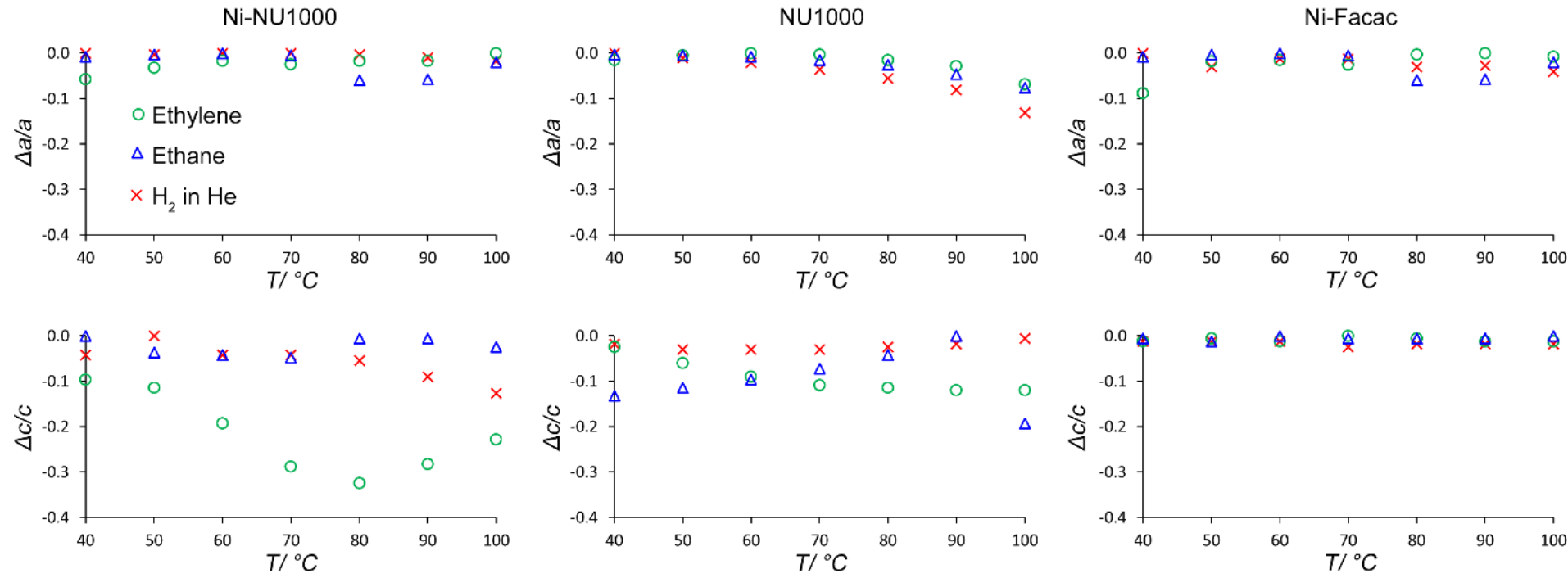

Figure S2. Temperature-dependence of the lattice dimensions under different gas atmospheres

Table S1. Coefficients of thermal expansion $\left(30-80^{\circ} \mathrm{C}\right)$ in different gas atmospheres

\begin{tabular}{|c|c|c|c|c|c|c|}
\hline & \multicolumn{2}{|c|}{ NiNU1000 } & \multicolumn{2}{c|}{ NU1000 } & \multicolumn{2}{c|}{ NiFacac } \\
\hline & $\alpha_{\mathrm{a}}\left(\times 10^{-6} \mathrm{~K}^{-1}\right)$ & $\alpha_{\mathrm{a}}\left(\times 10^{-6} \mathrm{~K}^{-1}\right)$ & $\alpha_{\mathrm{a}}\left(\times 10^{-6} \mathrm{~K}^{-1}\right)$ & $\alpha_{\mathrm{c}}\left(\times 10^{-6} \mathrm{~K}^{-1}\right)$ & $\alpha_{\mathrm{a}}\left(\times 10^{-6} \mathrm{~K}^{-1}\right)$ & $\alpha_{\mathrm{c}}\left(\times 10^{-6} \mathrm{~K}^{-1}\right)$ \\
\hline Ethylene & $\mathbf{+ 1 1}$ & $-\mathbf{6 5}$ & 2 & -24 & 2 & $\sim 0$ \\
\hline Ethane & $\sim 0$ & $\sim 0$ & -4 & 20 & $\sim 0$ & $\sim 0$ \\
\hline $\mathrm{H}_{2}$ in He & $\sim 0$ & -5 & -8 & -4 & -4 & -4 \\
\hline
\end{tabular}




\section{Gas adsorption isotherms}

Low pressure ethylene and ethane adsorption experiments up to 1 bar were performed on each sample (Figures S3a-c and S4a-c) at selected temperatures $\left(25^{\circ} \mathrm{C}, 40^{\circ} \mathrm{C}\right.$, and $70{ }^{\circ} \mathrm{C}$ ). Each sample was treated at $120{ }^{\circ} \mathrm{C}$ for overnight under high vacuum on a Smart VacPrep from Micromeritics before each gas adsorption measurement. Measurements were done using a Micromeritics ASAP 2020 gas adsorption analyzer. Note: the maximum temperature accessed using the heater bath is $70{ }^{\circ} \mathrm{C}$.

To quantify the energetics of interaction between ethylene/ethane and MOFs, the isosteric heat of adsorption $\left(\mathrm{Q}_{\mathrm{st}}\right)$ over the entire studied range was calculated for NU-1000, Ni-NU1000, and Ni-FacacNU1000, using $\mathrm{C}_{2} \mathrm{H}_{4}$ and $\mathrm{C}_{2} \mathrm{H}_{6}$ isotherms at $25{ }^{\circ} \mathrm{C}, 40{ }^{\circ} \mathrm{C}$, and $70{ }^{\circ} \mathrm{C}$, applying Clausius-Clapeyron equation (Figures S3d and S4d). NU-1000 has similar heats of adsorption for both ethylene and ethane which is consistent with similarity of their binding distribution. For Ni-NU1000 and Ni-Facac, the ethane has higher heat of adsorption than ethylene.

(a)
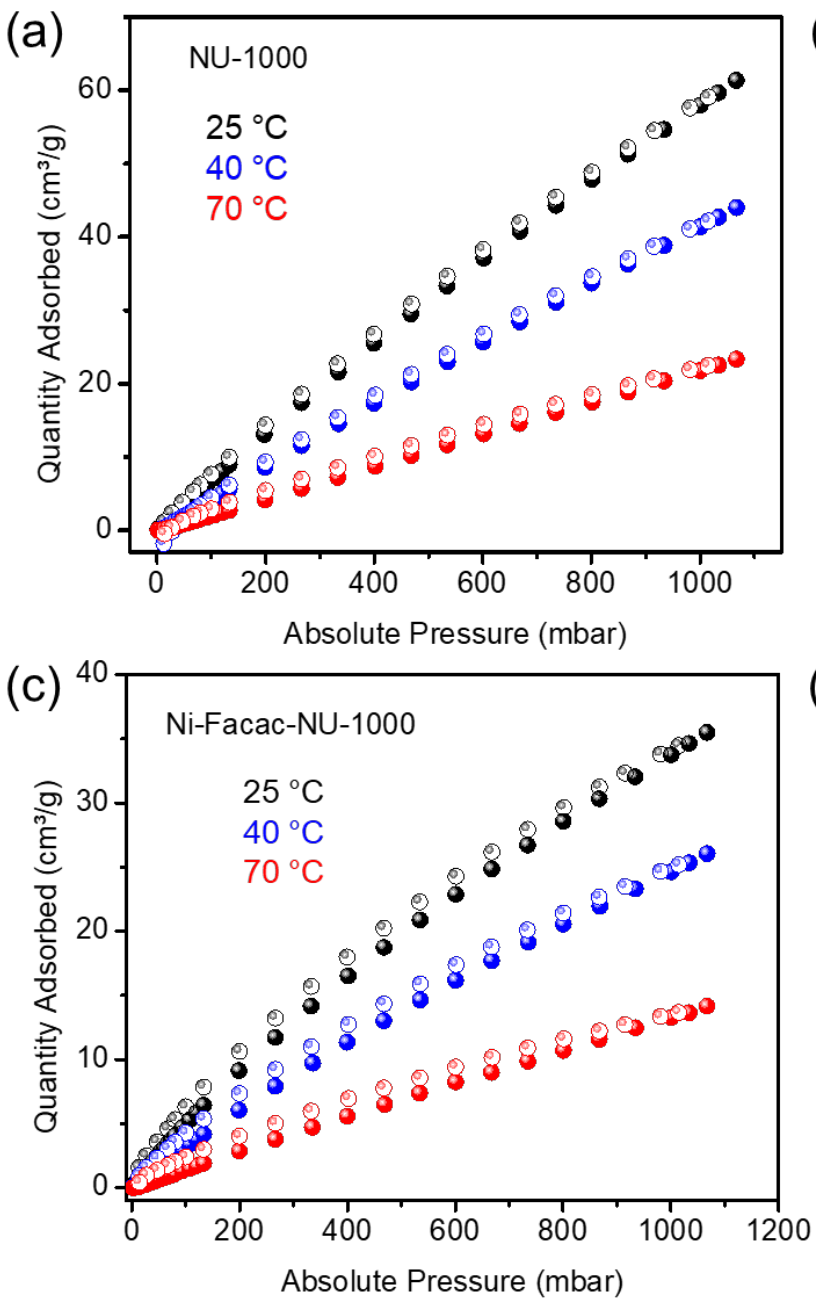
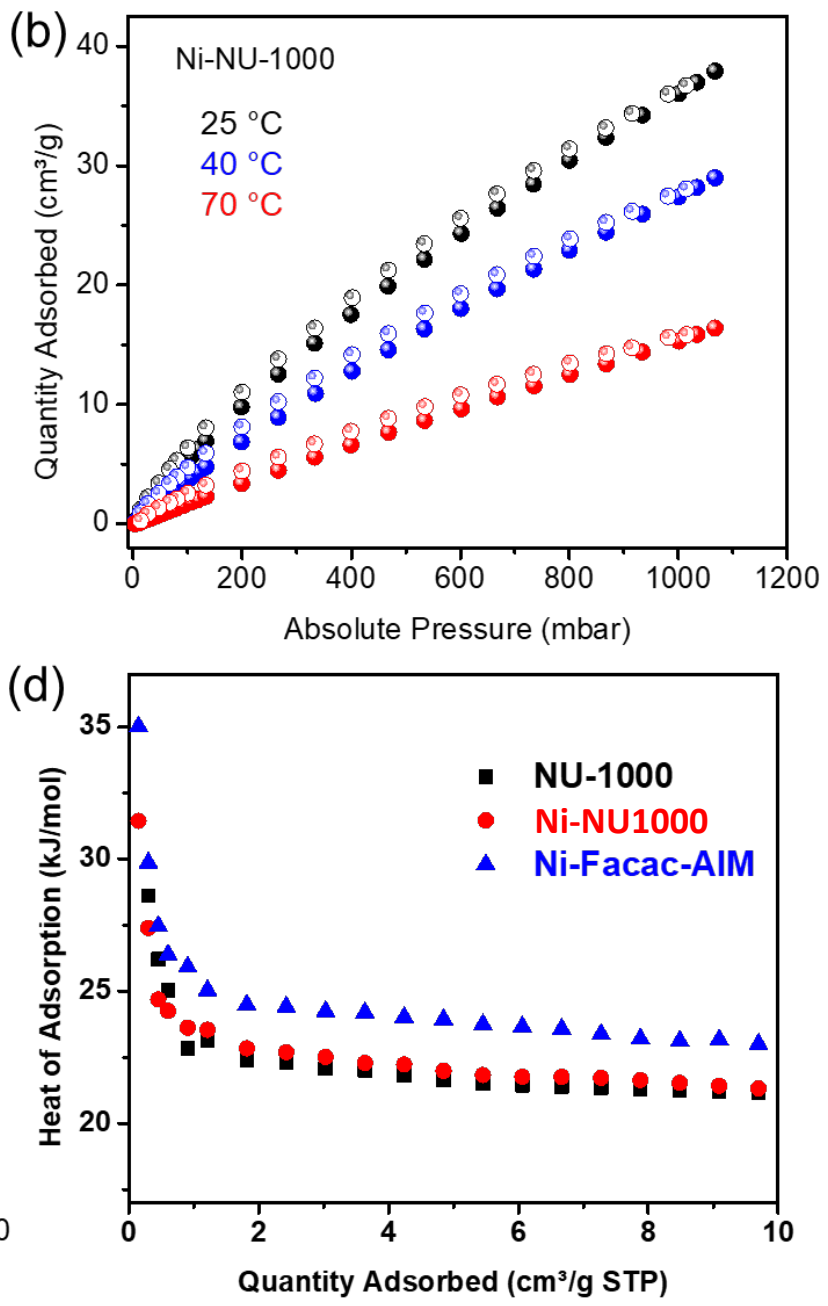

Figure S3. Ethylene adsorption for a) NU-1000, b) Ni-NU1000, and c) Ni-Facac; and (d) the $Q_{s t}$ calculated. 

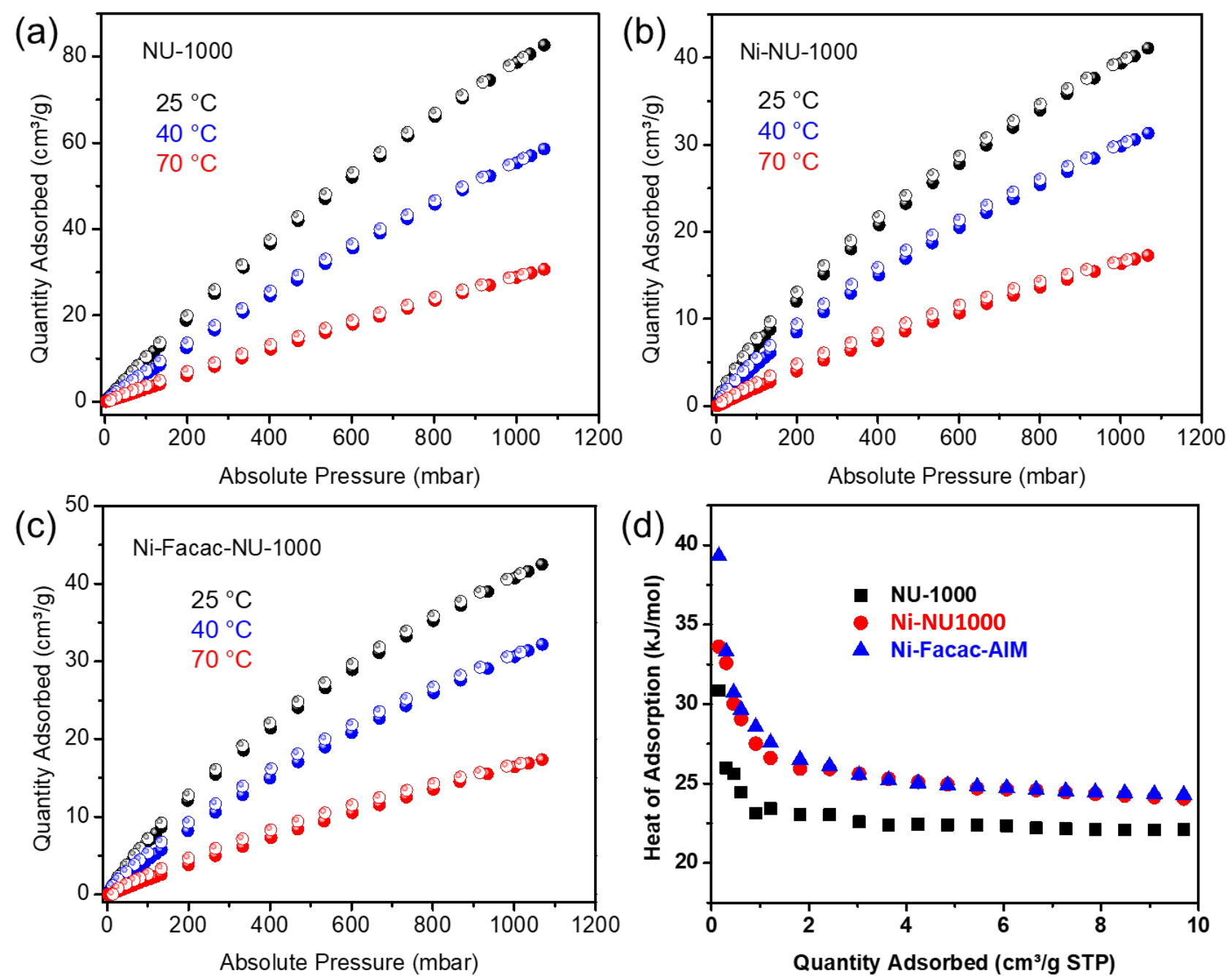

Figure S4. Ethane adsorption for a) NU-1000, b) Ni-NU1000, and c) Ni-Facac; and (d) the $Q_{s t}$ calculated. 


\section{Catalytic Ethylene Hydrogenation Activity}

Catalytic ethylene hydrogenation activity was recorded using a packed-bed flow reactor (Microactivity Efficient, MAE). The products were analyzed using an Agilent 7890A GC system equipped with an FID detector using an Agilent J\&W GC column (GS-Alumina, $30 \mathrm{~m} \times 0.535 \mathrm{~mm}$ ) and the quantity of each gas evaluated based on the integrated areas. Gas-phase hydrogenation catalysis used 3\% ethylene in $\mathrm{Ar}, 5 \%$ $\mathrm{H}_{2}$ in Ar and UHP Ar. The catalyst ( $25 \mathrm{mg} \mathrm{Ni}$-Facac or $\sim 3 \mathrm{mg}$ Ni-NU1000, 4:6 Ni/Zr, diluted with $200 \mathrm{mg}$ of $\mathrm{SiO}_{2}$ ) was packed on quartz wool in a stainless reactor. The reaction temperature was controlled with a $\mathrm{K}$ type thermocouple at the top of the catalyst bed. For the catalyst pretreatment during the hydrogenation process, the temperature was ramped at $10^{\circ} \mathrm{C} / \mathrm{min}$ to a final temperature of $200^{\circ} \mathrm{C}$ under a flow of $200 \mathrm{~mL} / \mathrm{min} 5 \% \mathrm{H}_{2} / \operatorname{Ar}$ (1.5 bar pressure) for $2 \mathrm{~h}$. It was then cooled down to the desired temperature $\left(100^{\circ} \mathrm{C}\right)$ under a flow of $200 \mathrm{~mL} / \mathrm{min} \mathrm{Ar}$ within one hour. For the catalysis, the ratio of flow rates between the two reacting gases and $\mathrm{Ar}$ was kept constant for all the experiments (ethylene : $\mathrm{H}_{2}: \mathrm{Ar}$ $=1: 2: 3$ ).

By varying the space velocity of ethylene, the turnover frequency (TOF) for Ni-Facac catalyzed ethylene hydrogenation was determined to be $(3.6 \pm 0.5) \times 10^{-4} \mathrm{~s}^{-1}$ on a per-nickel-atom basis assuming every $\mathrm{Ni}$ is catalytic active (Figure S5a), making it two orders of magnitude less active than Ni-NU1000 ((5.0 \pm 0.3$) \times$ $10^{-2} \mathrm{~s}^{-1}$ ) under the same conditions (Figure $\mathrm{S} 5 \mathrm{~b}$ ).
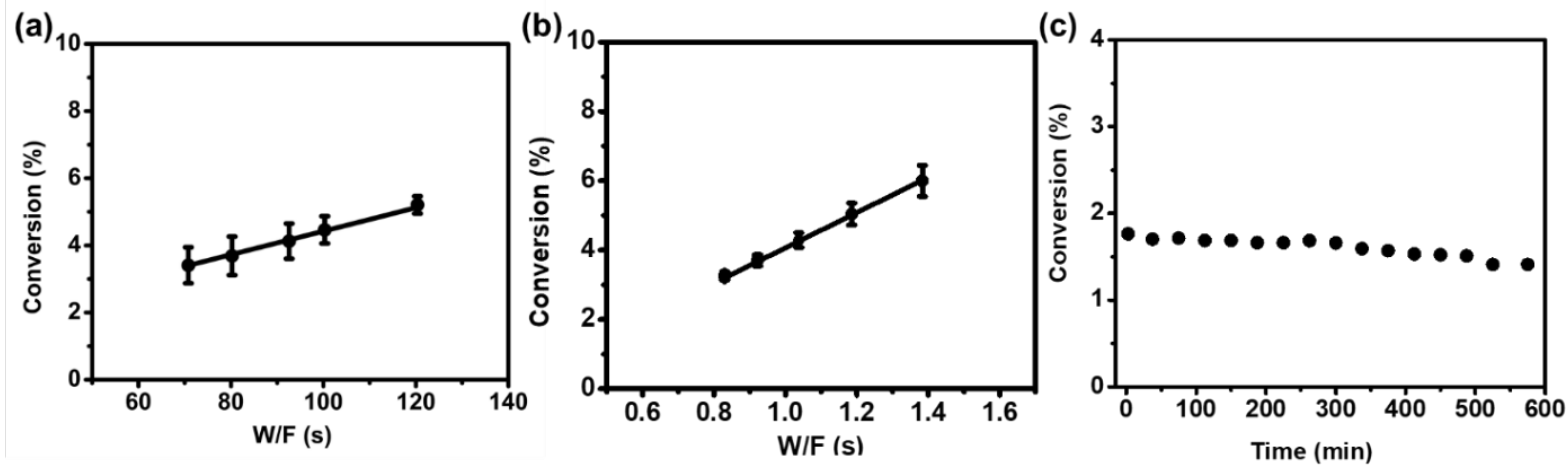

Figure S5. Catalytic conversion of ethylene to ethane by a) activated Ni-Facac; and b) activated Ni-NU1000; (c) Temporal assessment of stability of activated Ni-Facac-NU-1000 for ethylene hydrogenation. 


\section{Computational Approach}

The adsorption of ethane and ethylene was studied by considering a periodic model of the NU-1000 framework. DFT calculations with periodic boundary conditions were carried out using the $\mathrm{PBE}^{11}$ exchange-correlation functional as implemented in the VASP program package. ${ }^{12-13}$ The weak van der Waals interactions are calculated semi-empirically following the Grimme's (-D3) method. ${ }^{14}$ Core electrons were described within the projector augmented wave (PAW) scheme. For $C$ and $O$, the $2 s$ and $2 p$ electrons are treated explicitly; for $\mathrm{Zr}$, the $4 \mathrm{~s}, 4 \mathrm{p}, 5 \mathrm{~s}$ and $4 \mathrm{~d}$ electrons are included (together with the $\mathrm{Zr}$ _sv PAW pseudopotential). The core radii for $\mathrm{Zr}, \mathrm{O}, \mathrm{C}$ and $\mathrm{H}$ were 2.50, 1.52, 1.50 and 1.10 a.u. respectively. The valence electrons were described by a plane wave basis set with a kinetic energy cut-off of $520 \mathrm{eV}$. In all optimizations, the cell parameters and ionic positions of the framework atoms were kept fixed and only

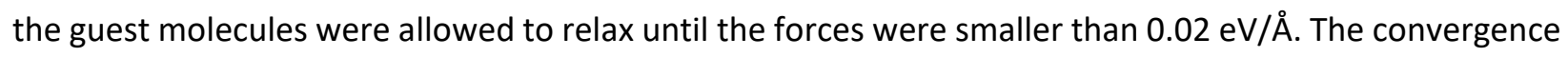
criteria for the electronic step were set to $10^{-6} \mathrm{eV}$. The computational details were also used in our previous works establishing the proton topology and studying the structural distortions of NU-1000. ${ }^{15-16}$ The periodic structure of the NU-1000 is also taken from these works.

Enthalpies were calculated based on the quantum mechanical harmonic-oscillator approximation for the vibrational partition function assuming that the guest molecules have a partial gas pressure of $0.1 \mathrm{Atm}$. The rotational and translational partition functions are neglected when the guests are adsorbed in the solid. Due to the partial geometry optimizations, a partial hessian was also calculated, by considering only the atoms of the guest molecules. Harmonic force constants were calculated numerically by displacing each atom by a negative and positive step-size of $0.01 \AA$ in all three cartesian directions. The convergence criterion for the electronic step was set to $10^{-6} \mathrm{eV}$ for the calculation of harmonic force constants. Due to the nature of the weakly bound complexes, the frequencies are expected to be anharmonic and thus not possible to be described within the harmonic-oscillator approximation. This effect has also been shown in other works on the adsorption of small molecules in zeolites and MOFs. ${ }^{17-19}$ These frequencies are affiliated with relative rotational motions of ethane and ethylene inside the pores of the framework. In many cases, low lying or even imaginary frequencies were calculated. It was not possible to eliminate these modes, even by applying stricter optimization criteria or re-optimizing the structures. For that reason, we decided to replace the values of imaginary and low-lying frequencies (with magnitude less than $50 \mathrm{~cm}^{-1}$ ), with values of $50 \mathrm{~cm}^{-1}$ for the calculation of enthalpies. 


\section{Computational Results}

Several initial conformations were considered according to the experimental observations: i) the center, ii) the edge (i.e: the pyrene linker), and iii) the corner (or pore window). We also considered a fourth adsorption site: the small pore that is defined as the space between the two $\mathrm{Zr}_{6}$-based nodes. To simplify the problem, we chose to study the adsorption of the hydrocarbons with the NU-1000 that retains the original proton topology. ${ }^{5}$

The results on binding energies and adsorption enthalpies agree qualitatively with the experimental observations. For both gases, the strongest binding site is predicted to be in the pore window, followed by the pyrene linker. All results are presented in Tables S2 \& S3. We have identified many close-lying minima indicating that the potential energy surface for the adsorption is very flat, thus many degenerate binding positions exist for the center, edge and corner site. The flatness and anharmonicity of the potential energy surface are also reflected in the imaginary frequencies that are calculated within the harmonic-oscillator approximation and are also shown in Tables S2 \& S3.

For ethane, the relative energy difference of the corner and edge sites is $2-3 \mathrm{kJmol}^{-1}$ (after accounting for temperature effects in enthalpies), with the corner site having adsorption enthalpies of $\sim 16 \mathrm{kJmol}^{-1}$, and the edge site of $\sim 13 \mathrm{kJmol}^{-1}$, while for ethylene their relative energy difference is calculated to be significantly larger: $6-9 \mathrm{kJmol}^{-1}$, with adsorption enthalpies of $\sim 20 \mathrm{kJmol}^{-1}$ and the edge site of $\sim 11 \mathrm{kJmol}^{-}$

1. The center of triangular pore is predicted to be the weakest binding site among all, with very low adsorption enthalpies. Interestingly, calculations indicate that the small pore is a stronger binding than the center of the triangular channel. We calculate adsorption enthalpies of $\sim 8 \mathrm{kJmol}^{-1}$ for both ethane and ethylene.

However, the center site may become important in higher loadings. To prove this, we calculate several configurations with 3 and 4 ethylene molecules adsorbed inside the triangular channel. The most important are presented in Figure S6. For the first case, we calculate an average binding energy of -20.8 $\mathrm{kJmol}^{-1}$ per ethylene molecule, whereas the binding of one single ethylene is $-17.5 \mathrm{kJmol}^{-1}$. Thus, there is an extra stabilization of $\sim 3.3 \mathrm{kJmol}^{-1}$ between each adsorbed ethylene with the other two neighboring species. Similar effect is calculated for the adsorption of 4 ethylene molecules: 3 near the corner sites and 1 in the center. In this case, the total binding energy for the four molecules is $-66.1 \mathrm{kJmol}^{-1}$. After decomposing the individual interactions, we find that total binding energy of the three species at the corner sites with the framework is $-53.0 \mathrm{kJmol}^{-1}$, whereas the fourth has a binding energy of $-4.4 \mathrm{kJmol}^{-1}$ in the absence of the other three. Thus, there is an additional stabilization energy of $8.7 \mathrm{kJmol}^{-1}$ that arises from the interactions of the fourth ethylene with the other three co-adsorbed species. Therefore, the lateral interactions become very pronounced for the center adsorption site. 
Table S2: Enthalpy and energy results (in $\mathrm{kJmol}^{-1}$ ) for Ethane adsorption in NU-1000. $\Delta \Delta E$ (ads) is the relative binding energy with respect to the most stable adsorption site.

\begin{tabular}{|c|c|c|c|c|c|c|c|c|}
\hline \multirow[t]{3}{*}{ Site } & & \multirow[t]{3}{*}{ Imag Freqs } & \multirow[t]{3}{*}{$\Delta \mathrm{E}$ (ads) } & \multirow[t]{3}{*}{$\Delta \Delta \mathrm{E}$ (ads) } & \multicolumn{4}{|l|}{$\Delta \mathbf{H}$} \\
\hline & & & & & \multicolumn{4}{|c|}{ (Temperature, K) } \\
\hline & & & & & 0 & 313 & 343 & 373 \\
\hline \multirow[t]{7}{*}{ Corner } & 1 & $2:-46,-88$ & -20.0 & +1.1 & -16.9 & -14.4 & -13.9 & -13.4 \\
\hline & $2 A$ & $2:-56,-84$ & -20.1 & +1.0 & -17.5 & -14.7 & -14.2 & -13.7 \\
\hline & 2B & $2:-39,-93$ & -20.1 & +1.0 & -17.1 & -14.6 & -14.1 & -13.7 \\
\hline & $2 \mathrm{C}$ & 1: -22 & -20.4 & +0.7 & -17.3 & -14.9 & -14.4 & -13.9 \\
\hline & 3A & $3:-49,-61,-108$ & -20.1 & +1.0 & -17.9 & -14.9 & -14.4 & -13.9 \\
\hline & 3B & $3:-30,-41,-71$ & -20.7 & +0.4 & -18.5 & -15.4 & -14.9 & -14.4 \\
\hline & $3 C$ & $3:-40,-58,-73$ & -21.1 & +0 & -18.8 & -15.8 & -15.3 & -14.8 \\
\hline \multirow[t]{5}{*}{ Edge } & $1 \mathrm{~A}$ & $2:-61,-87$ & -18.4 & +2.7 & -15.7 & -12.8 & -12.3 & -11.9 \\
\hline & 1B & $3:-23,-55,-107$ & -18.4 & +2.7 & -15.4 & -12.8 & -12.3 & -11.8 \\
\hline & $2 A$ & $3:-45,-72,-96$ & -18.7 & +2.4 & -16.3 & -13.3 & -12.8 & -12.3 \\
\hline & 2B & $2:-65,-84$ & -18.7 & +2.4 & -16.2 & -13.2 & -12.7 & -12.3 \\
\hline & $2 C$ & 1: -8 & -19.2 & +1.9 & -15.9 & -13.5 & -13.1 & -12.6 \\
\hline \multirow[t]{3}{*}{ Center } & 1 & $\begin{array}{l}4:-35,-75,-87,- \\
100\end{array}$ & -7.1 & +14.0 & & & & \\
\hline & $2 A$ & $2:-42,-49$ & -8.0 & +13.1 & -5.2 & -2.5 & -2.0 & -1.5 \\
\hline & 2B & $2:-13,-91$ & -8.0 & +13.1 & -5.3 & -2.5 & -2.1 & -1.6 \\
\hline \multirow[t]{4}{*}{ Small Pore } & $1 \mathrm{~A}$ & $3:-20,-26,-85$ & -10.9 & +10.2 & -8.8 & -8.2 & -8.0 & -7.7 \\
\hline & $2 A$ & $3:-59,-69,-95$ & -12.7 & +8.8 & -11.1 & -7.6 & -7.1 & -6.6 \\
\hline & 2B & $\begin{array}{l}4:-42,-52,-62,- \\
79\end{array}$ & -12.7 & +8.8 & -10.6 & -7.5 & -7.1 & -6.6 \\
\hline & $2 C$ & $\begin{array}{l}5:-19,-42,-68,- \\
97,-119\end{array}$ & -13.2 & +7.9 & -11.2 & -8.1 & -7.6 & -7.1 \\
\hline
\end{tabular}


Table S3: Enthalpy and energy results (in $\mathrm{kJmol}^{-1}$ ) for Ethylene adsorption in NU-1000. $\triangle \Delta E$ (ads) is the relative binding energy with respect to the most stable adsorption site

\begin{tabular}{|c|c|c|c|c|c|c|c|c|}
\hline \multirow[t]{3}{*}{ Site } & & \multirow[t]{3}{*}{ Imag Freqs } & \multirow[t]{3}{*}{$\Delta \mathrm{E}$ (ads) } & \multirow[t]{3}{*}{$\Delta \Delta \mathrm{E}$ (ads) } & \multicolumn{4}{|l|}{$\Delta \mathrm{H}$} \\
\hline & & & & & \multicolumn{4}{|c|}{ (Temperature, K) } \\
\hline & & & & & 0 & 313 & 343 & 373 \\
\hline \multirow[t]{7}{*}{ Corner } & $1 \mathrm{~A}$ & $\begin{array}{l}3:-39,-97,- \\
103\end{array}$ & -16.6 & +8.7 & -14.1 & -11.3 & -10.8 & -10.3 \\
\hline & 1B & 1: -110 & -24.7 & +0.5 & -21.4 & -19.1 & -18.6 & -18.2 \\
\hline & $1 \mathrm{C}$ & & -25.2 & +0.0 & -21.9 & -19.6 & -19.2 & -18.7 \\
\hline & $2 A$ & 2: $-86,-109$ & -17.2 & +7.9 & -14.7 & -11.8 & -11.4 & -10.9 \\
\hline & 2B & $2:-44,-71$ & -14.8 & +10.4 & -12.7 & -9.5 & -9.0 & -8.5 \\
\hline & $3 A$ & $2:-44,-76$ & -17.4 & +7.8 & -14.0 & -11.8 & -11.3 & -10.8 \\
\hline & 3B & & -17.5 & +7.7 & & & & \\
\hline \multirow[t]{6}{*}{ Edge } & 1 & $2:-42,-58$ & -15.5 & +9.7 & -11.9 & -9.8 & -9.3 & -8.9 \\
\hline & $2 A$ & $2:-87,-93$ & -14.8 & +10.4 & -11.8 & -9.2 & -8.7 & -8.3 \\
\hline & 2B & $2:-72,-99$ & -14.9 & +10.3 & -11.5 & -9.1 & -8.7 & -8.2 \\
\hline & $3 A$ & $2:-31,-70$ & -15.1 & +10.1 & -11.5 & -9.2 & -8.8 & -8.3 \\
\hline & 3B & 1: -51 & -15.1 & +10.1 & -11.4 & -9.3 & -8.9 & -8.4 \\
\hline & 4 & $2:-29,-96$ & -16.9 & +8.3 & -13.3 & -11.2 & -10.7 & -10.2 \\
\hline \multirow[t]{3}{*}{ Center } & $1 \mathrm{~A}$ & $3:-4,-62,-94$ & -5.5 & +19.7 & -3.3 & -0.1 & 0.4 & 0.9 \\
\hline & 1B & $\begin{array}{l}4:-27,-102,- \\
109,-134\end{array}$ & -5.8 & +19.4 & -3.7 & -0.4 & 0.1 & 0.6 \\
\hline & 2 & $\begin{array}{l}3:-36,-72,- \\
140\end{array}$ & -4.7 & +20.5 & -1.9 & 0.9 & 1.4 & 1.9 \\
\hline Small Pore & 1 & $2:-14,-67$ & -13.6 & +11.6 & -10.3 & -8.1 & -7.6 & -7.2 \\
\hline
\end{tabular}




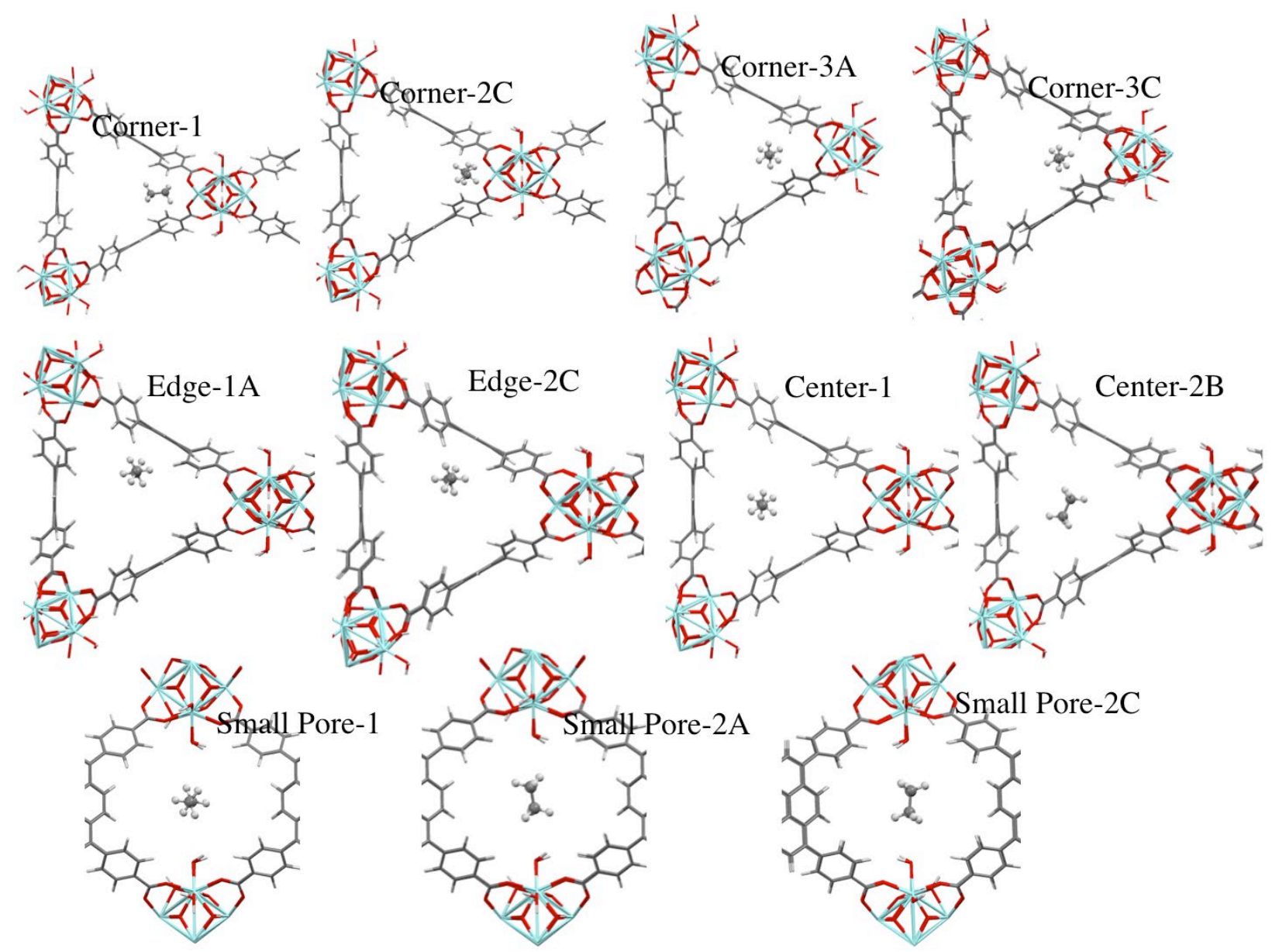

Figure S6. Some representative conformations for the adsorption of ethane in NU-1000. These correspond to the binding sites in Table S1. 


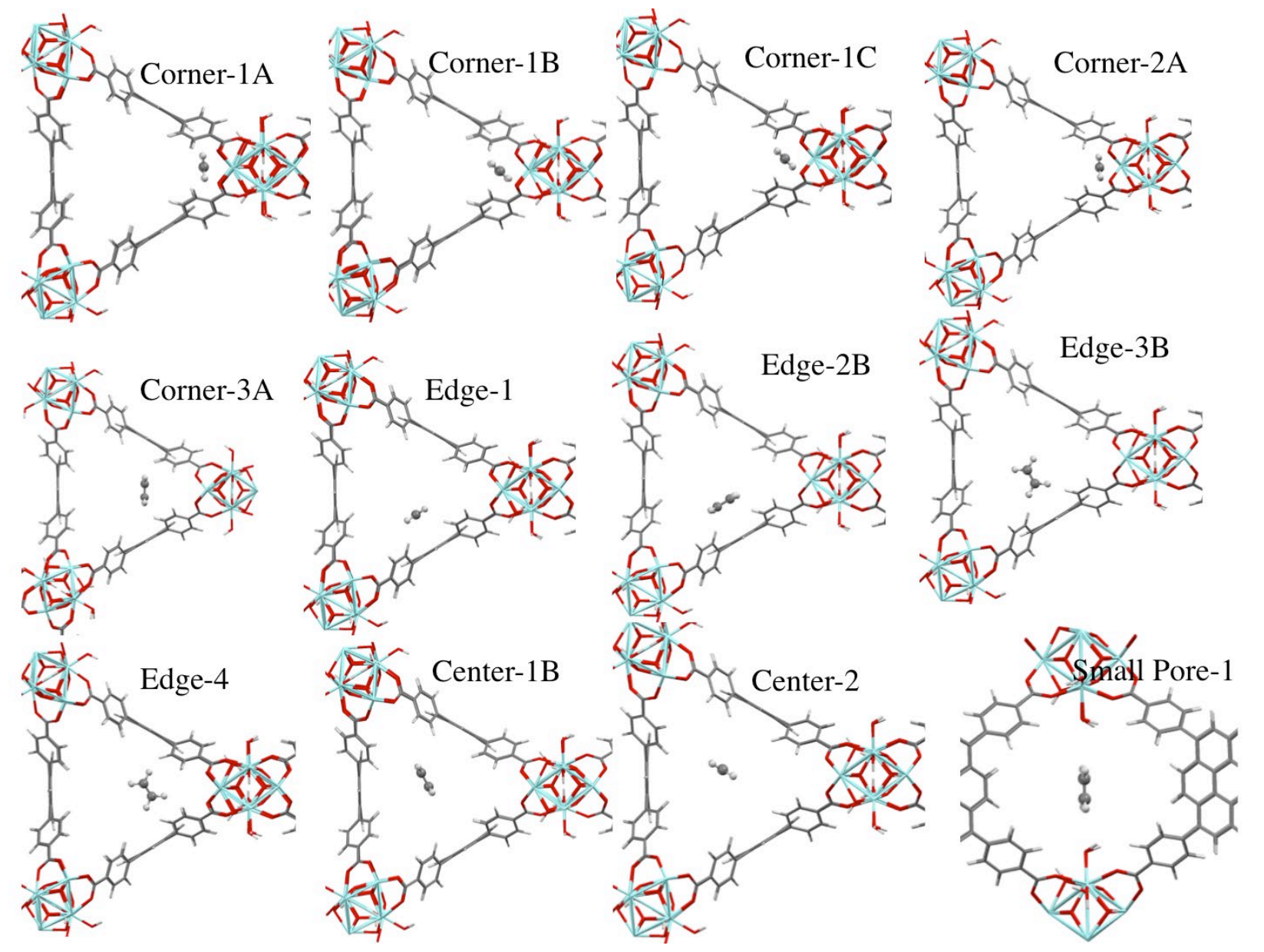

Figure S7. Some representative conformations for the adsorption of ethylene in NU-1000. These correspond to the binding sites in Table S2. 

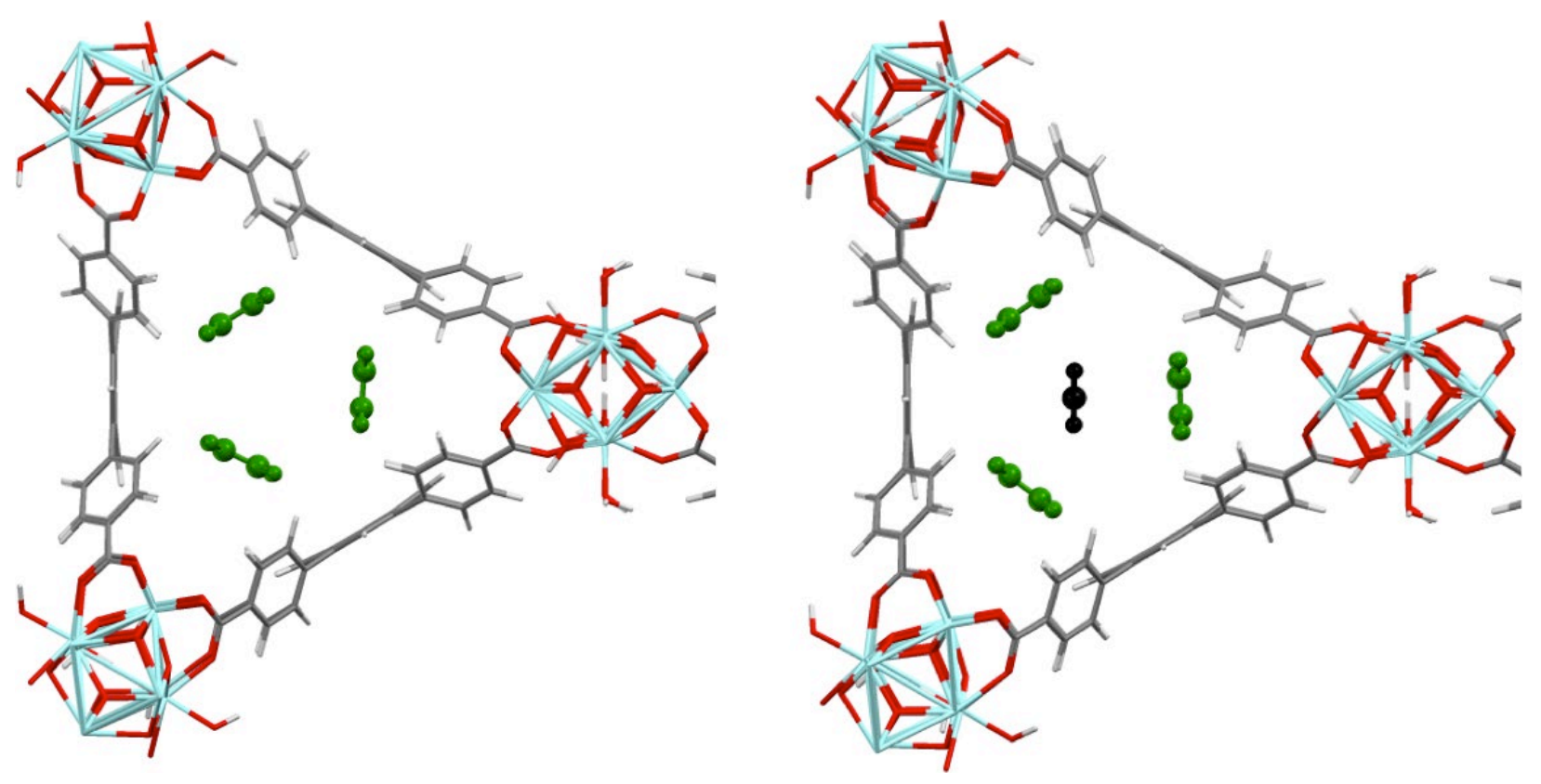

Figure S8. Adsorption of multiple (3 and 4) ethylene molecules in NU-1000. Ethylene molecules adsorbed at the Corner site are presented in green color, whereas the molecule at the Center site in black. 


\section{References:}

1. Mondloch, J. E.; Bury, W.; Fairen-Jimenez, D.; Kwon, S.; DeMarco, E. J.; Weston, M. H.; Sarjeant, A. A.; Nguyen, S. T.; Stair, P. C.; Snurr, R. Q.; Farha, O. K.; Hupp, J. T., Vapor-Phase Metalation by Atomic Layer Deposition in a Metal-Organic Framework. J. Am. Chem. Soc. 2013, 135 (28), 10294-10297.

2. Li, Z.; Schweitzer, N. M.; League, A. B.; Bernales, V.; Peters, A. W.; Getsoian, A.; Wang, T. C.; Miller, J. T.; Vjunov, A.; Fulton, J. L.; Lercher, J. A.; Cramer, C. J.; Gagliardi, L.; Hupp, J. T.; Farha, O. K., Sintering-Resistant SingleSite Nickel Catalyst Supported by Metal Organic Framework. J. Am. Chem. Soc. 2016, 138 (6), 1977-1982.

3. Chupas, P. J.; Chapman, K. W.; Kurtz, C.; Hanson, J. C.; Lee, P. L.; Grey, C. P., A versatile sample-environment cell for non-ambient X-ray scattering experiments. J. Appl. Crystallogr. 2008, 41, 822-824.

4. Toby, B. H.; Von Dreele, R. B., GSAS-II: the genesis of a modern open-source all purpose crystallography software package. J. Appl. Crystallogr. 2013, 46 (2), 544-549.

5. Petříček, V.; Dušek, M.; Palatinus, L., Crystallographic Computing System JANA2006: General features. Z. Kristallogr. Cryst. Mater. 2014, 229 (5), 345.

6. McCusker, L. B.; Baerlocher, C., Using electron microscopy to complement X-ray powder diffraction data to solve complex crystal structures. Chem. Commun. 2009, (12), 1439-1451.

7. Yakovenko, A. A.; Reibenspies, J. H.; Bhuvanesh, N.; Zhou, H.-C., Generation and applications of structure envelopes for porous metal-organic frameworks. J. Appl. Crystallogr. 2013, 46 (2), 346-353.

8. $\quad$ Chen, Y.-P.; Liu, Y.; Liu, D.; Bosch, M.; Zhou, H.-C., Direct Measurement of Adsorbed Gas Redistribution in Metal-Organic Frameworks. J. Am. Chem. Soc. 2015, 137 (8), 2919-2930.

9. Yakovenko, A. A.; Wei, Z.; Wriedt, M.; Li, J.-R.; Halder, G. J.; Zhou, H.-C., Study of Guest Molecules in MetalOrganic Frameworks by Powder X-ray Diffraction: Analysis of Difference Envelope Density. Cryst. Growth Des. 2014, 14 (11), 5397-5407.

10. Platero-Prats, A. E.; League, A. B.; Bernales, V.; Ye, J. Y.; Gallington, L. C.; Vjunov, A.; Schweitzer, N. M.; Li, Z. Y.; Zheng, J.; Mehdi, B. L.; Stevens, A. J.; Dohnalkova, A.; Balasubramanian, M.; Farha, O. K.; Hupp, J. T.; Browning, N. D.; Fulton, J. L.; Camaioni, D. M.; Lercher, J. A.; Truhlar, D. G.; Gagliardi, L.; Cramer, C. J.; Chapman, K. W., Bridging Zirconia Nodes within a Metal-Organic Framework via Catalytic Ni-Hydroxo Clusters to Form Heterobimetallic Nanowires. J. Am. Chem. Soc. 2017, 139 (30), 10410-10418.

11. Perdew, J. P.; Burke, K.; Ernzerhof, M., Generalized Gradient Approximation Made Simple. Phys. Rev. Lett. 1996, 77 (18), 3865-3868.

12. Kresse, G.; Furthmüller, J., Efficient Iterative Schemes for Ab Initio Total-Energy Calculations Using a PlaneWave Basis Set. Phys. Rev. B 1996, 54, 11169.

13. Kresse, G.; Furthmüller, J., Efficiency of ab-initio total energy calculations for metals and semiconductors using a plane-wave basis set. Computational Materials Science 1996, 6 (1), 15-50.

14. Grimme, S.; Antony, J.; Ehrlich, S.; Krieg, H., A Consistent and Accurate Ab Initio Parametrization of Density Functional Dispersion Correction (DFT-D) for the 94 Elements H-Pu. J. Chem. Phys. 2010, 132, 154104.

15. Planas, N.; Mondloch, J. E.; Tussupbayev, S.; Borycz, J.; Gagliardi, L.; Hupp, J. T.; Farha, O. K.; Cramer, C. J., Defining the Proton Topology of the Zr6-Based Metal-Organic Framework NU-1000. J. Phys. Chem. Lett. 2014, 5 (21), 3716-3723.

16. Platero-Prats, A. E.; Mavrandonakis, A.; Gallington, L. C.; Liu, Y.; Hupp, J. T.; Farha, O. K.; Cramer, C. J.; Chapman, K. W., Structural Transitions of the Metal-Oxide Nodes within Metal-Organic Frameworks: On the Local Structures of NU-1000 and UiO-66. J. Am. Chem. Soc. 2016, 138 (12), 4178-4185.

17. Piccini, G.; Sauer, J., Effect of Anharmonicity on Adsorption Thermodynamics. J. Chem. Theory Comput. 2014, 10, 2479-2487.

18. Piccini, G.; Alessio, M.; Sauer, J.; Zhi, Y.; Liu, Y.; Kolvenbach, R.; Jentys, A.; Lercher, J. A., Accurate Adsorption Thermodynamics of Small Alkanes in Zeolites. Ab initio Theory and Experiment for H-Chabazite. J. Phys. Chem. C 2015, 119 (11), 6128-6137.

19. Kundu, A.; Piccini, G.; Sillar, K.; Sauer, J., Ab Initio Prediction of Adsorption Isotherms for Small Molecules in Metal-Organic Frameworks. J. Am. Chem. Soc. 2016, 138 (42), 14047-14056. 